\title{
GALAXY EVOLUTION FROM QSO ABSORPTION-LINE SELECTED SAMPLES
}

\author{
MARK DICKINSON \\ STScI, 3700 San Martin Dr., Baltimore MD 21218, USA \\ AND
}

CHARLES C. STEIDEL

Caltech Astronomy, MS 105-24, Pasadena CA 91125, USA

\section{QSO Absorption Lines and Galaxies}

Although a connection between certain classes of QSO absorption line systems and gas associated with galaxies was hypothesized long ago, the first systematic evidence supporting this was provided by Bergeron \& Boissé (1991). Observing QSOs with known MgII absorption lines at $z_{\mathrm{abs}}<z_{\mathrm{QSO}}$, they identified galaxies near the QSO sightline and spectroscopically confirmed that their redshifts matched those of the MgII absorption doublet.

Since that time, we have been carrying out surveys aimed at (1) characterizing the nature of the galaxies selected by this method, and (2) using these galaxies to study the evolution of the field galaxy population at high redshift. We refer to our sample as one "selected by gas cross-section," since a MgII rest-frame equivalent width $W_{0}>0.3 \AA$ is essentially equivalent to a neutral hydrogen column density $\mathrm{N}(\mathrm{HI})>10^{17} \mathrm{~cm}^{-2}$. For the purposes of studying field galaxy evolution, this is useful primarily because the selection depends only on a robust and easily measured rest frame property $\left(W_{0}(\mathrm{MgII})\right)$, and not on any observed frame characteristic such as apparent magnitude, color, surface brightness, etc. The method is thus free of many potential biases which affect deep magnitude-limited redshift surveys (although it may be subject to its own!). Moreover, once the gas halo cross-sections are understood (see below), the resulting sample is volumelimited, which is greatly advantageous when studying luminosity functions and the like. If the nature of absorption-selected galaxies can be firmly established, then comparison between our samples and those from the deep 
redshift surveys (such as the CFRS sample discussed by Lilly in the present volume) may lead to new insights about galaxy evolution.

Our first survey studied MgII absorption systems at $0.2<z<1.0$, and is essentially finished, with nearly complete imaging identification of candidate absorbers and spectroscopic redshift confirmation for $\sim 80 \%$ of these candidates. We are presently pushing for $100 \%$ redshift completeness using the W.M. Keck $10 \mathrm{~m}$ telescope. The second survey covers the range $1<z<2$ and is now underway using infrared and optical imaging from KPNO, Palomar and Keck, as well as selected spectroscopic follow-up from Keck. Space limitations prevent more than a cursory summary of results from these surveys, and no elaboration of the methods, procedures, data, or analyses is offered. For these, the reader is directed to previously published descriptions, including Steidel \& Dickinson 1992, Steidel, Dickinson \& Persson 1994, Steidel \& Dickinson 1995, and Steidel 1995.

For the range $0.2<z<1.0$, we find that all galaxies with luminosities $L_{K}>0.05 L_{K}^{*}$ are potentially $\mathrm{MgII}$ absorbers, provided that they fall within a particular impact parameter of the QSO sightline. This impact parameter (effectively, the gaseous halo radius) scales weakly with luminosity as $R_{\text {halo }}=38 h^{-1}\left(L_{K} / L_{K}^{*}\right)^{0.15} \mathrm{kpc}$, (where $h=H_{0} / 100 \mathrm{~km} / \mathrm{s} / \mathrm{Mpc}$ ). Conversely, we find no cases of bright intervening galaxies within this impact parameter limit which do not produce absorption. The only interlopers have turned out to be dwarfs, mostly very blue. Apparently, the MgII systems are dominated by "big" galaxies (within a few magnitudes of $L^{*}$ ). The "faint blue galaxies" which dominate deep number counts do not contribute appreciably to the gas cross-section of the Universe at $\mathrm{N}(\mathrm{HI})>10^{17} \mathrm{~cm}^{-2}$. The $K$-band luminosity function of the absorbing galaxies follows a Schechterlike distribution with $L^{*}$ (at $\left.\langle z\rangle \approx 0.65\right)$ indistinguishable from the presentday value, but with a high normalization consistent with values measured from the deep field galaxy surveys (e.g. the CFRS) at similar redshifts.

Interestingly, the halo radius vs. luminosity scaling is better behaved when computed with $K$-band magnitudes (instead of optical photometry). Moreover, we see no relation between a galaxy's color and its nature as an absorber. The absorbers span the range of normal galaxy colors from flatspectrum "Magellenic irregulars" to red ellipticals. There is no evidence for color evolution in the absorber population out to $z=1$, although Lilly et al. (1995) have shown that our results are not formally inconsistent with the evolution of the field galaxy population seen in the CFRS. Evidently, to first order, the presence of an extended gaseous halo does not depend on the current star formation rate in a galaxy, but rather on the luminosity of its older stellar population. Because $L_{K}$, for evolved galaxies, roughly traces the total stellar mass, we might therefore suppose that the halo diameter primarily reflects the mass of the galaxy. 
Our extension of this survey to the redshift regime $1<z<2$ has only begun, but the preliminary indications are that nothing is dramatically different from the situation at $z<1$ - the galaxy luminosities, colors, impact parameters, and space densities are roughly the same. Overall, this suggests that the "big" (massive?) galaxy population has been, for the most part, remarkably stable over a very long span of cosmic time. The apparent absence of luminosity evolution might seem to contradict expectations for simple passive evolution of stellar populations. However, this may be interpreted as implying a roughly constant star formation rate with redshift when averaged across the absorber population.

\section{MgII Absorber Morphologies}

Recently, we have begun obtaining HST WFPC2 images of MgII absorbers in order to evaluate their morphologies and to measure their orientations and inclination angles relative to the quasar line of sight. In Cycle 4 we imaged 3C 336, a $z=0.92$ quasar with the largest number of foreground absorbers (5!) along any single line of sight in our survey. In Cycle 5, we are imaging 12 additional fields. All images are taken through the F702W filter $\left(\lambda_{\text {eff }} \approx 7000 \AA\right)$.

Figure 1 shows a montage of the absorbers imaged to date. Most are fairly ordinary galaxies spanning the range of normal Hubble Types, from bulge-dominated systems (e.g. the $z=0.318$ and 0.660 absorbers) to latetype disks (e.g. the $z=0.442$ and 0.723 galaxies). A few exotic objects are also found - the $z=0.525$ absorber looks like the highly elongated, peculiar galaxies seen in many deep HST images and emphasized by Cowie et al. 1995. This galaxy, however, is extremely red, suggesting that it is a highly reddened, edge-on disk - extinction may account for its odd morphology. Several absorbers are highly inclined (e.g. the $z=0.891$ galaxy), reinforcing other evidence that MgII absorption arises from halo material rather than from gas in the disk. The few $z>1$ galaxies we have imaged look somewhat peculiar, but this may only reflect the fact that the $R$-band WFPC2 images sample their emitted-frame ultraviolet continuum.

M.D. would like to thank the organizers for their hospitality and travel support, as well as STScI for additional funds. This work was partially supported by NASA/STScI grants GO-5304 and GO-5984.

\section{References}

Bergeron, J., and Boissé, P. 1991, A\&A, 243, 344.

Cowie, L.L., Hu, E.M., and Songaila, A. 1995, Nature, 377, 603.

Lilly, S.J., Tresse, L., Hammer, F., Crampton, D., and Le Fèvre, O. 1995, ApJ. (in press).

Steidel, C.C., and Dickinson, M. 1992, ApJ., 394, 81. 
Steidel, C.C., Dickinson, M., and Persson, S.E. 1994, ApJ., 437, L75.

Steidel, C.C., and Dickinson, M. 1995, in Wide Field Spectroscopy 8 the Distant Universe, eds. S. Maddox and A. Aragón-Salamanca, World Scientific, p. 349.

Steidel, C.C., in QSO Absorption Lines, ed. G. Meylan, Springer, p. 139.

\begin{tabular}{|c|c|c|c|}
\hline$z=0.318$ & $z=0.368$ & $z=0.442$ & $z=0.472$ \\
\hline$z=0.525$ & $z=0.553$ & $z=0.640 ?$ & $z=0.660$ \\
\hline$z=0.723$ & $z=0.798$ & $z=0.838$ & $z=0.891$ \\
\hline$z=1.201$ & $z=1.282 ?$ & $z=1.457$ & $z=1.526$ \\
\hline
\end{tabular}

Figure 1: Montage of HST WFPC2 images of MgII absorbing galaxies, arranged by redshift. Each panel is 4 arcsec on a side. Cases where the galaxy redshifts have not been confirmed spectroscopically are indicated with a question mark. 